\title{
Calculation of the Green's function in the scattering region for first-principles electron-transport simulations
}

\author{
Yoshiyuki Egami, ${ }^{1,{ }^{*}}$ Shigeru Tsukamoto $\odot,{ }^{2}$ and Tomoya Ono ${ }^{3}$ \\ ${ }^{1}$ Division of Applied Physics, Faculty of Engineering, Hokkaido University, Sapporo, Hokkaido 060-8628, Japan \\ ${ }^{2}$ Peter Grünberg Institut \& Institute for Advanced Simulation, Forschungszentrum Jülich and JARA, D-52425 Jülich, Germany \\ ${ }^{3}$ Department of Electrical and Electronic Engineering, Kobe University, Kobe, Hyogo 657-8501, Japan
}

(Received 2 May 2020; accepted 14 December 2020; published 14 January 2021)

\begin{abstract}
We propose a first-principles method of efficiently evaluating electron-transport properties of very long systems. Implementing the recursive Green's function method and the shifted conjugate gradient method in the transport simulator based on real-space finite-difference formalism, we can suppress the increase in the computational cost, which is generally proportional to the cube of the system length to a linear order. This enables us to perform the transport calculations of double-walled carbon nanotubes (DWCNTs) with 196608 atoms. We find that the conductance spectra exhibit different properties depending on the periodicity of doped impurities in DWCNTs and they differ from the properties for systems with less than 1000 atoms.
\end{abstract}

DOI: 10.1103/PhysRevResearch.3.013038

\section{INTRODUCTION}

One-dimensional materials such as nanowires and nanotubes, which have unique electronic properties due to the quantum confinement effect, are expected to be applied to electronic and spintronic devices, optoelectronic circuits, and biosensors [1-7]. In recent years, large-scale electron-transport calculations have been indispensable for designing the functionality of electronic devices. Although first-principles calculations based on the density functional theory (DFT) [8] allow us to accurately evaluate electrontransport properties of atomic systems, their target is typically limited to small systems because of the heavy computational cost arising from calculating the Green's functions, which is intrinsic cubic scaling with system size. Limited-scale system is sufficient to simulate simple systems, such as locally perturbed bulk regions or interfaces. However, it is not suitable for simulating complex systems, such as bulk with a realistic defect density, amorphous structure, and interfaces with lattice mismatches between different crystalline materials. Thus far, to circumvent this restriction, transport calculations for large systems containing several thousands of atoms have been performed within atomic-basis formalism and tight-binding (TB) formalism based on DFT [9-12].

On the other hand, real-space finite-difference (RSFD) formalism is also recognized as suitable for large-scale calculations requiring high computational accuracy [13-20]. In this formalism, a Hamiltonian matrix is expressed as a block

\footnotetext{
*Corresponding author: y_egami@eng.hokudai.ac.jp

Published by the American Physical Society under the terms of the Creative Commons Attribution 4.0 International license. Further distribution of this work must maintain attribution to the author(s) and the published article's title, journal citation, and DOI.
}

tridiagonal matrix with sparse matrix elements, which is a great advantage in solving equations by iterative methods, and the sparsity of the matrix can be controlled by the order of the finite-difference approximation, $N_{\mathrm{f}}$. The computational accuracy can be systematically improved by narrowing the grid spacing. Moreover, the RSFD formalism has a high affinity to massively parallel architectures. In order to improve the efficiency of parallel computing, it is important not only to optimize the arithmetic algorithm, but also to equalize the computational load and data communication in each process. In the RSFD formalism, in the spatial domain decomposition, the whole space is divided into subspaces of equal size, so that the computational load depending on the number of grid points can be equalized. In addition, although wave functions in adjacent subspaces assigned to other processes are required for stencil calculations, only wave functions at grid points near the boundary between subspaces need to be communicated, which is sufficiently small in data volume. so the amount of data communication between processes can be uniform and small. Therefore it is possible to perform efficient parallel computing with uniform computational load and data communication.

Fujimoto and Hirose developed the overbridging boundary matching (OBM) method based on the RSFD formalism [13] by exploiting the advantages for electron-transport calculations, where a whole system is divided along the $z$ direction (see Fig. 1) into three regions: the left electrode, the transition region and the right electrode. The electron-transport calculation refers only to subsets of the Green's function matrix of the transition region. In the OBM method, the subsets are calculated efficiently using a shifted conjugate-gradient (SCG) solver [16] since the Hamiltonian matrix in the RSFD formalism is sparse. In the SCG solver, the computational cost for calculating the subsets is $\mathcal{O}\left(N_{E} N_{x y}^{3} N_{z}\right)$ when the algorithm proposed by Takayama et al. [21] is adopted, where $N_{x y}$ and $N_{z}$ are the numbers of grid points in the $x y$ plane and the $z$ 


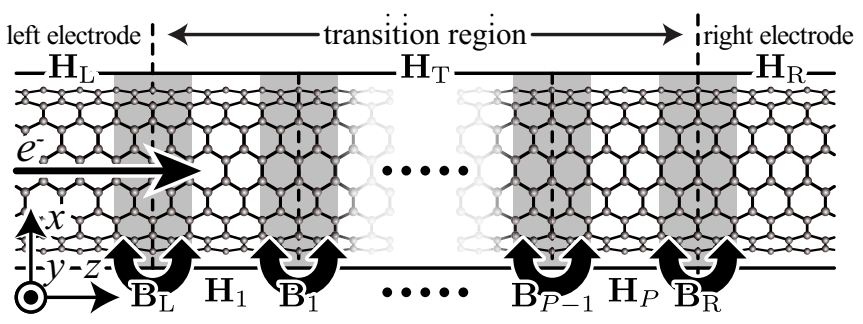

FIG. 1. Schematic representation of a junction system. The system is partitioned into one transition region and left and right semi-infinite electrodes, whose Hamiltonians are denoted by $\mathbf{H}_{\mathrm{T}}, \mathbf{H}_{\mathrm{L}}$, and $\mathbf{H}_{\mathrm{R}}$, respectively. $\mathbf{B}_{\mathrm{L}(\mathrm{R})}$ denotes the interaction between adjoining small parts. The transition region consists of $P$ parts. The Hamiltonians are denoted by $\mathbf{H}_{i}$ for $i=1, \ldots, P$, and the interactions between the adjoining parts by $\mathbf{B}_{i}$ for $i=1, \ldots, P-1$.

direction, respectively, and $N_{E}$ denotes the number of energy values $E$ to be treated at once by the SCG method. For systems with a large $N_{z}$, the computational cost for matrix-vector operations, which is $\mathcal{O}\left(N_{x y}^{3} N_{z}^{2}\right)$, becomes dominant [22]. Although the maximum order of the computational cost is reduced from $\mathcal{O}\left(N_{x y}^{3} N_{z}^{3}\right)$ to $\mathcal{O}\left(N_{x y}^{3} N_{z}^{2}\right)$ by the OBM method, the transport calculation has room for further reducing the computational cost to handle more realistic and longer one-dimensional systems.

In this paper, we propose an efficient computational procedure based on RSFD formalism to evaluate the electrontransport properties of long systems containing more than 100000 atoms under the zero temperature and zero bias limits in the steady state without accuracy deterioration. We verify the computational accuracy and performance of the proposed method. Additionally, to exemplify the efficiency of the proposed procedure we demonstrate large-scale electrontransport calculations for double-walled carbon nanotubes (DWCNTs) composed of 196608 atoms, which make, to the best of our knowledge, the largest system in the first-principles electron-transport calculation.

The rest of this paper is composed as follows. Section II presents the theoretical procedure used to construct the Green's function matrix of a scattering region with a long dimension based on the recursive Green's function (RGF) method [23] within the framework of the RSFD formalism. Section III examines a computational accuracy and efficiency of our method and reveals the transport properties of impuritydoped DWCNTs is influenced by the length of systems and the arrangement of impurities. Section IV provides the conclusions. Appendix A demonstrates the computational cost of matrix-vector operations for calculating the Green's function matrices by SCG method. Appendices B-D describe some of the mathematical techniques used in this paper.

\section{COMPUTATIONAL SCHEME}

Thouless and Kirkpatrick proposed an efficient calculation of subsets of a Green's function matrix for a transition region within TB formalism as the RGF method. The RGF method constructs a transition region by arranging multiple parts along the $z$ direction as illustrated in Fig. 1, and calculates only necessary subsets by recursively combining the Green's function matrices of the adjoining small parts one by one. Since the calculation of the Green's function matrices for the small parts is less costly than that for the extended transition region, the RGF method is capable of handling large systems and is widely used for calculating the transport properties of atomic-basis models and TB models [24-30].

Now, we discuss the advantage of the RGF method in transport calculations within RSFD formalism. The transport properties are evaluated using the Green's function of the transition region suspended between semi-infinite electrodes, $\mathbf{G}_{\mathrm{T}}$, which is constructed by the Green's function associated with the truncated transition region, $\mathcal{G}_{\mathrm{T}}$, and the self-energy terms of the electrodes [15]. The Green's function matrix $\mathcal{G}_{\mathrm{T}}=\left(E \mathbf{I}_{\mathrm{T}}-\mathbf{H}_{\mathrm{T}}\right)^{-1}$, in which $\mathbf{H}_{\mathrm{T}}$ is the truncated Hamiltonian matrix of the extended transition region consisting of $P$ parts (see Fig. 1), is expressed within the framework of RSFD formalism using the norm-conserving pseudopotentials (NCPPs) as

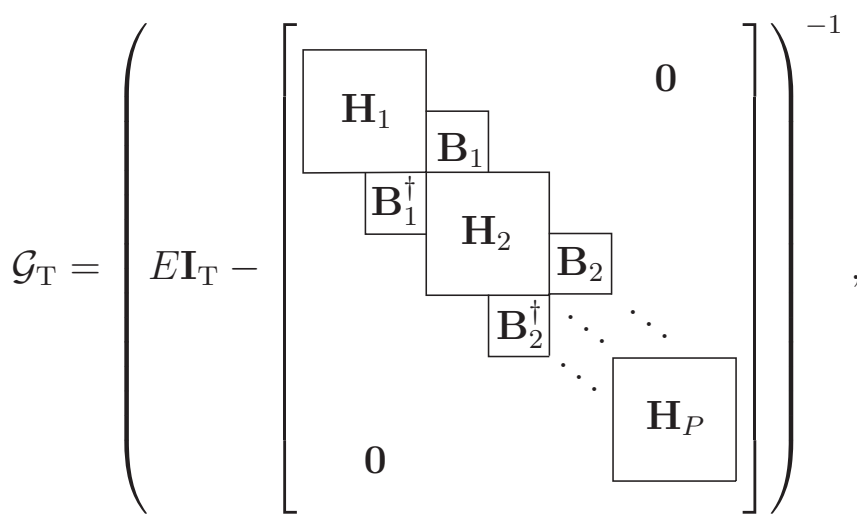

where $\mathbf{H}_{i}$ is the truncated Hamiltonian matrix for the transition region of the $i$ th part. For simplicity, we assume that all $\mathbf{H}_{i}(i=1, \ldots, P)$ have the same dimension, the interactions between the second- and further-neighboring parts are zero, and the nonzero matrix, $\mathbf{B}_{i}$, represents the interaction between the adjoining parts. We define the dimensions of $\mathbf{B}_{i}$ and $\mathbf{H}_{i}$ as $M_{i} \times N_{i}$ and $N_{x y} N_{z}^{(\text {part })}$, respectively, with $N_{z}^{(\text {part })}=N_{z} / P ; \mathbf{I}_{\mathrm{T}}$ denotes an $N_{x y} N_{z}$-dimensional identity matrix.

Since the Hamiltonian matrix of RSFD formalism is highly sparse, it is more advantageous to calculate the subsets of Green's function matrix of the transition region by iterative solvers than it is to compute the entire Green's function by direct inversions of the Hamiltonian matrix. According to Ref. [15], the electron-transport properties can be estimated using submatrices located at the four corners of the Green's function matrix, $\mathcal{G}_{\mathrm{T}}$. Here, we propose an algorithm for reproducing the submatrices of the entire Green's function required for electron-transport calculations by combining the subsets of the Green's function of adjoining parts.

We defined the Green's function matrix associated with the truncated Hamiltonian composed of the parts numbered from $i$ to $j$ as $\mathcal{G}_{i: j}$. Now we will combine the two adjoining parts to derive the Green's function matrix associated with the truncated Hamiltonian composed of the $i$ th and $(i+1)$ th parts 
(see Fig. 1), $\mathcal{G}_{i: i+1}$, which is written as

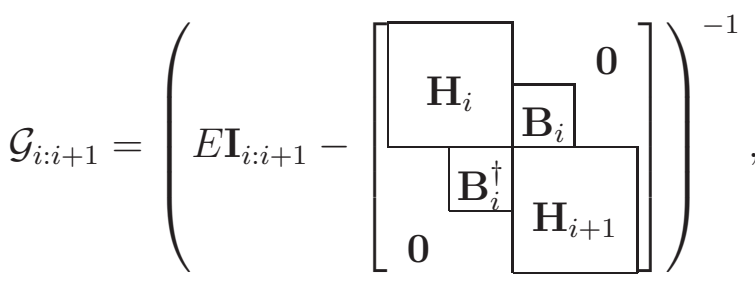

where $\mathcal{G}_{i: i+1}$ is the $2 N_{x y} N_{z}^{(\text {part })}$-dimensional matrix and the submatrices, $\mathbf{H}_{i}$ and $\mathbf{H}_{i+1}$, represent the truncated Hamiltonian matrices of the $i$ th and $(i+1)$ th parts, respectively. $\mathbf{I}_{i: i+1}$ denotes an identity matrix with a dimension of $\mathcal{G}_{i: i+1}$. We assume that the Green's function matrix associated with $\mathbf{H}_{i(i+1)}$ is already calculated as $\mathcal{G}_{i: i(i+1: i+1)}=\left(E-\mathbf{H}_{i(i+1)}\right)^{-1}$ [31]. We need only the submatrices located at the upper-left, upperright, lower-left, and lower-right corners of $\mathcal{G}_{i: i+1}$, which are denoted by $\mathcal{G}_{i: i+1}^{\mathrm{UL}}, \mathcal{G}_{i: i+1}^{\mathrm{UR}}, \mathcal{G}_{i: i+1}^{\mathrm{LL}}$, and $\mathcal{G}_{i: i+1}^{\mathrm{LR}}$, respectively. Here, a definition is necessary before we discuss this further. To indicate a specific block in a $2 \times 2$ partitioned matrix, following the quadrant notion in the coordinate geometry [32], we hereafter refer the blocks anticlockwise from the upper-right block as quadrants I, II, III, and IV as

$$
\left[\begin{array}{c|c}
\text { II } & \text { I } \\
\hline \text { III } & \text { IV }
\end{array}\right] \text {. }
$$

For example, the upper-left block is referred to as quadrant II.

Let us first calculate $\mathcal{G}_{i: i+1}^{\mathrm{UL}}$. According to Appendix B, the quadrant II of $\mathcal{G}_{i: i+1}$ is expressed as

$$
\mathcal{G}_{i: i+1}^{\mathrm{II}}=\left(E \mathbf{I}_{i}-\mathbf{H}_{i}+\left[\begin{array}{cc}
\mathbf{0} & \mathbf{0} \\
\mathbf{0} & -\mathbf{B}_{i} \mathcal{G}_{i+1: i+1}^{\mathrm{UL}} \mathbf{B}_{i}^{\dagger}
\end{array}\right]\right)^{-1},
$$

where $\mathcal{G}_{i+1: i+1}^{\mathrm{UL}}$ represents an $N_{i}$-dimensional square submatrix located at the upper-left corner of $\mathcal{G}_{i+1: i+1}$, and $\mathbf{I}_{i}$ denotes an identity matrix with the dimension of $\mathbf{H}_{i}$. It should be noted that an $N_{i-1}$-dimensional square submatrix located at the upper-left corner of $\mathcal{G}_{i: i+1}^{\mathrm{II}}$ corresponds with $\mathcal{G}_{i: i+1}^{\mathrm{UL}}$. Therefore, according to Appendix $\mathrm{C}, \mathcal{G}_{i: i+1}^{\mathrm{UL}}$ can be analytically derived as

$$
\begin{aligned}
\mathcal{G}_{i: i+1}^{\mathrm{UL}}= & \mathcal{G}_{i: i}^{\mathrm{UL}}+\mathcal{G}_{i: i}^{\mathrm{UR}} \mathbf{B}_{i} \mathcal{G}_{i+1: i+1}^{\mathrm{UL}} \mathbf{B}_{i}^{\dagger} \\
& \times\left[\mathbf{I}_{M_{i}}-\mathcal{G}_{i: i}^{\mathrm{LR}} \mathbf{B}_{i} \mathcal{G}_{i+1: i+1}^{\mathrm{UL}} \mathbf{B}_{i}^{\dagger}\right]^{-1} \mathcal{G}_{i: i}^{\mathrm{LL}},
\end{aligned}
$$

where $\mathbf{I}_{M_{i}}$ denotes an $M_{i}$-dimensional identity matrix.

In the same way, $\mathcal{G}_{i: i+1}^{\mathrm{LR}}$ can be derived from the quadrant IV of $\mathcal{G}_{i: i+1}$, which is expressed as

$$
\mathcal{G}_{i: i+1}^{\mathrm{IV}}=\left(E \mathbf{I}_{i+1}-\mathbf{H}_{i+1}+\left[\begin{array}{cc}
-\mathbf{B}_{i}^{\dagger} \mathcal{G}_{i+1: i+1}^{\mathrm{LR}} \mathbf{B}_{i} & \mathbf{0} \\
\mathbf{0} & \mathbf{0}
\end{array}\right]\right)^{-1},
$$

where $\mathcal{G}_{i+1: i+1}^{\mathrm{LR}}$ represents an $M_{i}$-dimensional square submatrix located at the lower-right corner of $\mathcal{G}_{i+1: i+1}$, and $\mathbf{I}_{i+1}$ denotes an identity matrix with a dimension of $\mathbf{H}_{i+1} \cdot \mathcal{G}_{i: i+1}^{\mathrm{LR}}$ is found at the lower-right corner of $\mathcal{G}_{i: i+1}^{\mathrm{IV}}$. According to Appendix D,
$\mathcal{G}_{i: i+1}^{\mathrm{LR}}$ can be analytically derived as

$$
\begin{aligned}
\mathcal{G}_{i: i+1}^{\mathrm{LR}}= & \mathcal{G}_{i+1: i+1}^{\mathrm{LR}}+\mathcal{G}_{i+1: i+1}^{\mathrm{LL}} \mathbf{B}_{i}^{\dagger} \mathcal{G}_{i: i}^{\mathrm{LR}} \mathbf{B}_{i} \\
& \times\left[\mathbf{I}_{N_{i}}-\mathcal{G}_{i+1: i+1}^{\mathrm{UL}} \mathbf{B}_{i}^{\dagger} \mathcal{G}_{i: i}^{\mathrm{LR}} \mathbf{B}_{i}\right]^{-1} \mathcal{G}_{i+1: i+1}^{\mathrm{UR}},
\end{aligned}
$$

where $\mathbf{I}_{N_{i}}$ denotes an $N_{i}$-dimensional identity matrix.

Next, we calculated $\mathcal{G}_{i: i+1}^{\mathrm{UR}}$. Based on Appendix B and Eq. (2), the quadrant I of $\mathcal{G}_{i: i+1}$ is derived as

$$
\mathcal{G}_{i: i+1}^{\mathrm{I}}=-\mathcal{G}_{i: i+1}^{\mathrm{II}}\left[\begin{array}{ccc}
\mathbf{0} & \cdots & \mathbf{0} \\
\mathbf{B}_{i} \mathcal{G}_{i+1: i+1}^{\mathrm{UL}} & \cdots & \mathbf{B}_{i} \mathcal{G}_{i+1: i+1}^{\mathrm{UR}}
\end{array}\right] .
$$

Note that the last row block in the second matrix on the righthand side is the product of $\mathbf{B}_{i}$ and the submatrix composed of the first $N_{i}$ rows of $\mathcal{G}_{i+1: i+1}$. Since $\mathcal{G}_{i: i+1}^{\mathrm{UR}}$ is an $\left(N_{i-1} \times M_{i+1}\right)$ dimensional submatrix located at the upper-right corner of $\mathcal{G}_{i: i+1}^{\mathrm{I}}, \mathcal{G}_{i: i+1}^{\mathrm{UR}}$ is given as

$$
\mathcal{G}_{i: i+1}^{\mathrm{UR}}=-\mathcal{G}_{i: i+1}^{\mathrm{II}, \mathrm{UR}} \mathbf{B}_{i} \mathcal{G}_{i+1: i+1}^{\mathrm{UR}},
$$

where $\mathcal{G}_{i: i+1}^{\text {II,UR }}$ denotes an $\left(N_{i-1} \times M_{i}\right)$-dimensional submatrix located at the upper-right corner of $\mathcal{G}_{i: i+1}^{\mathrm{II}}$. As seen in Appendix $\mathrm{C}, \mathcal{G}_{i: i+1}^{\mathrm{II}, \mathrm{UR}}$ is analytically derived; hence, one can obtain $\mathcal{G}_{i: i+1}^{\mathrm{UR}}$ as

$$
\mathcal{G}_{i: i+1}^{\mathrm{UR}}=-\mathcal{G}_{i: i}^{\mathrm{UR}}\left[\mathbf{I}_{M_{i}}-\mathbf{B}_{i} \mathcal{G}_{i+1: i+1}^{\mathrm{UL}} \mathbf{B}_{i}^{\dagger} \mathcal{G}_{i: i}^{\mathrm{LR}}\right]^{-1} \mathbf{B}_{i} \mathcal{G}_{i+1: i+1}^{\mathrm{UR}} .
$$

In the same way, the quadrant III of $\mathcal{G}_{i: i+1}$ is expressed as

$$
\mathcal{G}_{i: i+1}^{\mathrm{III}}=-\mathcal{G}_{i: i+1}^{\mathrm{IV}}\left[\begin{array}{ccc}
\mathbf{B}_{i}^{\dagger} \mathcal{G}_{i: i}^{\mathrm{LL}} & \ldots & \mathbf{B}_{i}^{\dagger} \mathcal{G}_{i: i}^{\mathrm{LR}} \\
\mathbf{0} & \ldots & \mathbf{0}
\end{array}\right] .
$$

Note that the first row block in the second matrix on the right-hand side is the product of $\mathbf{B}_{i}^{\dagger}$ and the matrix composed of the last $M_{i}$ rows of $\mathcal{G}_{i: i}$. Since $\mathcal{G}_{i: i+1}^{\mathrm{LL}}$ is an $\left(M_{i+1} \times N_{i-1}\right)$ dimensional submatrix located at the lower-left corner of $\mathcal{G}_{i: i+1}^{\mathrm{III}}, \mathcal{G}_{i: i+1}^{\mathrm{LL}}$ is given as

$$
\mathcal{G}_{i: i+1}^{\mathrm{LL}}=-\mathcal{G}_{i: i+1}^{\mathrm{IV}, \mathrm{LL}} \mathbf{B}_{i}^{\dagger} \mathcal{G}_{i: i}^{\mathrm{LL}},
$$

where $\mathcal{G}_{i: i+1}^{\mathrm{IV}, \mathrm{LL}}$ denotes an $\left(M_{i+1} \times N_{i}\right)$-dimensional submatrix located at the lower-left corner of $\mathcal{G}_{i: i+1}^{\mathrm{IV}}$. As seen in Appendix $\mathrm{D}, \mathcal{G}_{i: i+1}^{\mathrm{IV}, \mathrm{LL}}$ is analytically derived; hence, one can obtain $\mathcal{G}_{i: i+1}^{\mathrm{LL}}$ as

$$
\mathcal{G}_{i: i+1}^{\mathrm{LL}}=-\mathcal{G}_{i+1: i+1}^{\mathrm{LL}}\left[\mathbf{I}_{N_{i}}-\mathbf{B}_{i}^{\dagger} \mathcal{G}_{i: i}^{\mathrm{LR}} \mathbf{B}_{i} \mathcal{G}_{i+1: i+1}^{\mathrm{UL}}\right]^{-1} \mathbf{B}_{i}^{\dagger} \mathcal{G}_{i: i}^{\mathrm{LL}} .
$$

Consequently, the submatrices located at the upper-left, upper-right, lower-left, and lower-right corners of $\mathcal{G}_{i: i+1}$ are given as Eqs. (5), (10), (13), and (7), respectively. As shown in these equations, we only need the four corners of $\mathcal{G}_{i: i}$, which are efficiently obtained by using the SCG method. In most cases, $M_{i}=N_{i}=N_{\mathrm{f}} N_{x y}$, with $N_{\mathrm{f}}$ being the order of the finite-difference approximation [33]. Repeating this procedure to connect the parts numbered from $i$ though to $j$, we can construct the four submatrices of a $(j-i+1) N_{x y} N_{z}^{\text {(part) }}$ dimensional matrix $\mathcal{G}_{i: j}$.

Now, we estimate the computational cost of the proposed method. We assume that $\mathbf{B}_{\mathrm{L}(\mathrm{R})}$ (see Fig. 1) and $\mathbf{B}_{i}$ for $i=1, \ldots, P-1$ are $M$-dimensional square matrices with $M=N_{\mathrm{f}} N_{x y}$. In the case of the RGF method based on the atomic-basis formalism, $\mathbf{H}_{\mathrm{T}}$ is dense; that is, $M$ is not sufficiently smaller than $N_{x y} N_{z}^{\text {(part) }}$. Therefore the iterative solvers 
are not more efficient than direct inversion, and there is not much benefit in using Eqs. (5), (10), (13), and (7) to reduce the computational cost. Furthermore, the overlap matrix is generally not any scalar matrix; hence, the SCG method cannot be used to solve the linear systems. However, the RGF algorithm must be compatible with RSFD formalism since $\mathbf{H}_{i}$ is a blockdiagonal matrix and a sparse matrix [14,33]. The proposed method can be implemented without negatively affecting the benefit of the SCG method, in which the matrix-vector operations for shifted energy points are omitted, and only the matrix elements of subsets are updated for shifted energy points during SCG iteration. The computational cost of the matrix-vector products with a sparse matrix increases linearly with $N_{z}$, and the number of SCG iterations is proportional to $N_{z}$ (see Appendix A). Therefore the computational cost for the extended transition region is reduced from $\mathcal{O}\left(N_{x y}^{3} N_{z}^{2}\right)=$ $\mathcal{O}\left(N_{x y}^{3}\left(N_{z}^{(\text {part })} P\right)^{2}\right)$ to $\mathcal{O}\left(N_{x y}^{3}\left(N_{z}^{(\text {part })}\right)^{2} P\right)$ using the RGF and the SCG methods since the cost is $\mathcal{O}\left(N_{x y}^{3}\left(N_{z}^{(\text {part })}\right)^{2}\right)$ for each of the $P$ parts; that is, the increase in computational cost can be suppressed linearly for $P$. Although the computational cost for calculating Eqs. (5), (10), (13), and (7) is $\mathcal{O}\left(P M^{3}\right)$, where $M=N_{\mathrm{f}} N_{x y}$, with $N_{\mathrm{f}}$ being sufficiently smaller than $N_{z}^{\text {(part) }}$. Consequently, further computational cost reduction for $N_{z}$ is achieved by making use of the advantages of the RGF method and SCG method within the RSFD formalism in comparison with the conventional procedure [15].

\section{ACCURACY TEST AND APPLICATIONS}

We evaluated the computational error between the conductance spectra using the Green's function matrices obtained by the proposed method and the conventional method [15] for the BN-doped $(4,4) @(8,8)$ DWCNT to verify that the proposed method does not deteriorate the computational accuracy. The unit cell contains 192 atoms, and boron and nitrogen atoms are substitutionally co-doped into the outer tube along the circumferential direction. The dimensions are $L_{x(y)}=19.5 \AA$ and $L_{z}^{\text {(part) }}=9.84 \AA$. For this verification, a supercell has twice the dimension in the $z$ direction that of the unit cell, that is, $L_{z}^{\text {(ext) }}=2 L_{z}^{\text {(part) }}$. Using the electronic structure calculation code RSPACE $[14,34]$ based on RSFD formalism within the DFT framework, the effective potential of the unit cell is calculated with $1 \times 1 \times 10 k$-points, $N_{x y}=80 \times 80$, $N_{z}^{(\text {part })}=40, N_{\mathrm{f}}=4$, the NCPPs [35,36], and the local-density approximation (LDA) [37]. The Green's function calculation is collectively performed using the SCG method with $N_{E}=41$. The $8192 \mathrm{CPU}$ cores on Intel®Xeon®Gold 6154 are assigned to perform parallel computing for the Green's function matrices. Figure 2 shows the conductance spectra of the supercell evaluated using the proposed method with $P=2$ and the conventional method. There are no notable errors more than $2.5 \times 10^{-7} \mathrm{G}_{0}$.

Finally, we compared the computational times in calculating the Green's function submatrices for several systems using the proposed method with those using the conventional method. Here the computational times are measured for systems in which (001) Si bulk is doped with B atoms. We consider a primitive unit cell of (001) Si bulk, which contains

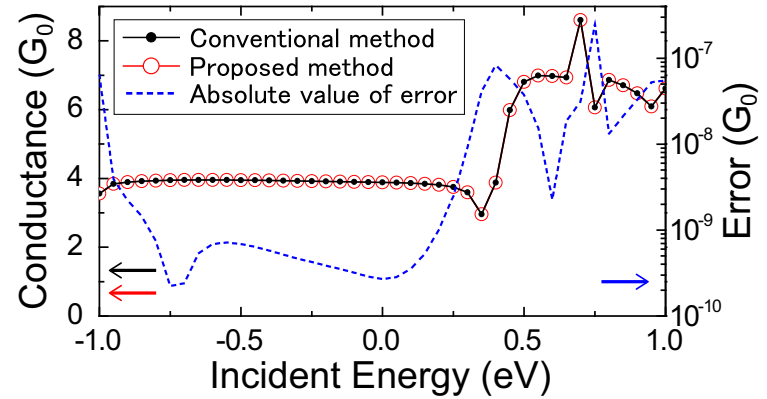

FIG. 2. Conductance spectra of DWCNT (solid lines) and computational error of the proposed method (dashed line).

four Si atoms and has the dimensions of $L_{x(y)}^{(\text {part })}=3.84 \AA$ and $L_{z}^{\text {(part) }}=5.43 \AA$. We measured the CPU time for calculating the submatrices of the Green's function matrix in a supercell with $L_{z}^{\text {(ext) }}=P L_{z}^{\text {(part) }}$, in which the $P$ unit cells are arranged along the $z$ direction, $(P=1,2,4$, and 8$)$, and any of the four $\mathrm{Si}$ atoms in each unit cell is randomly replaced with a $\mathrm{B}$ atom. The effective potential of the unit cell is calculated using the RSPACE code with $8 \times 8 \times 12 k$-points, $N_{x y}=12 \times 12$, $N_{z}^{\text {(part) }}=16, N_{\mathrm{f}}=4$, the NCPPs, and the LDA. We prepared small parts with $L_{x(y)}=n_{x(y)} \times L_{x(y)}^{(\text {part })}$ by duplicating the unit cell $n_{x(y)}$ times in the $x(y)$ direction with $n_{x}=n_{y}=1,2$, and 4 to verify the influence of the cross-sectional size on the CPU time. As summarized in Table I, the CPU time for calculating the four submatrices of $\mathcal{G}_{1: P}$ with $N_{E}=16$ using the proposed method increases in proportion to $P$. We confirmed that the proposed method enabled us to notably reduce the computational cost and avoid computational difficulties in handling long systems. Furthermore, one can see that the time ratio is not attenuated even in large cross-sectional systems. Since the proposed method has high weak-scaling efficiency for increasing $P$, the benefit of this method becomes more remarkable as the system size increases.

TABLE I. CPU time to obtain four submatrices of the Green's function matrix for B-doped (001) Si bulk models. The time ratio is evaluated as [calculating time + combining time]/[calculating time for $P=1]$. The calculations were performed with $1152 \mathrm{CPU}$ cores on Intel ${ }$ Xeon ${ }^{\circledR} C P U$ E5-2680.

\begin{tabular}{llrrr}
\hline \hline$\left(n_{x}, n_{y}\right)$ & $P$ & $\begin{array}{c}\text { Calculating } \\
\text { time (sec.) }\end{array}$ & $\begin{array}{c}\text { Combining } \\
\text { time (sec.) }\end{array}$ & $\begin{array}{l}\text { Time } \\
\text { ratio }\end{array}$ \\
\hline$(1,1)$ & 1 & 175 & - & 1.00 \\
& 2 & 352 & 19 & 2.12 \\
& 4 & 669 & 56 & 4.15 \\
& 8 & 1347 & 130 & 8.45 \\
$(2,2)$ & 1 & 13381 & - & 1.00 \\
& 2 & 26773 & 1457 & 2.11 \\
& 4 & 49171 & 4372 & 4.00 \\
& 8 & 98240 & 10206 & 8.10 \\
$(4,4)$ & 1 & 592191 & - & 1.00 \\
& 2 & 1183461 & 65451 & 2.11 \\
& 4 & 2159101 & 196408 & 3.98 \\
& 8 & 4317207 & 458096 & 8.06 \\
\hline \hline
\end{tabular}



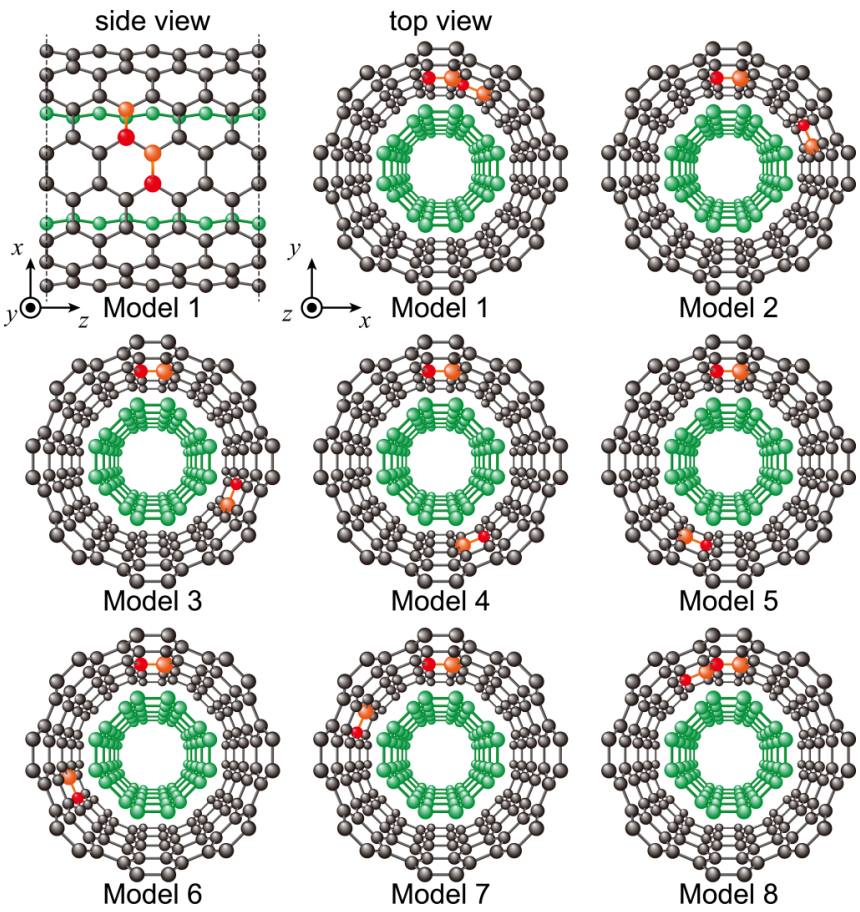

FIG. 3. Schematic view of the DWCNT unit-cell models. The grey and green spheres represent $\mathrm{C}$ atoms of the outer and inner carbon nanotubes, respectively, and the red and orange spheres represent $\mathrm{N}$ and $\mathrm{B}$ atoms, respectively. Dashed lines represent the unit cell boundaries.

With the above verifications, it is confirmed that electrontransport properties can be estimated efficiently without the deterioration of computational accuracy using the proposed method. As a practical demonstration of the method, we investigated the electron-transport properties of DWCNTs depending on the tube length. As illustrated in Fig. 3, eight DWCNT unit-cell models with different relative positions of the two $\mathrm{BN}$ dimers are prepared. Here, the transport properties are evaluated for a model in which the same unit cells (model 4) are periodically combined (periodic model) and in which different unit cells (Model 1-8) are randomly arranged (random model). For the periodic model, the transport calculations are performed for the DWCNTs with $P=1-1024$ to investigate the changes in the conductance spectrum according to the number of unit cells $P$ in the transition region. The numerical conditions and procedures to obtain the effective potential and the Green' function matrices of each unit cell are the same as those mentioned in the accuracy verification. Here, we ignore the electron-phonon couplings and evaluate the transport properties described in the ballistic regime [38].

As shown in Fig. 4, for systems with small $P$, the conductance quantization is observed at the low incident energy, where there are four conduction channels with a transmission probability of almost unity. In the low energy region, the incident electrons flow through the intra-states of the inner and outer tubes, as depicted in Fig. 5(a). Therefore there are high-transmission channels due to the innertube states that are insensitive to the scattering potential created by the $\mathrm{BN}$ dimers in the outer tube. However, the channel transmissions

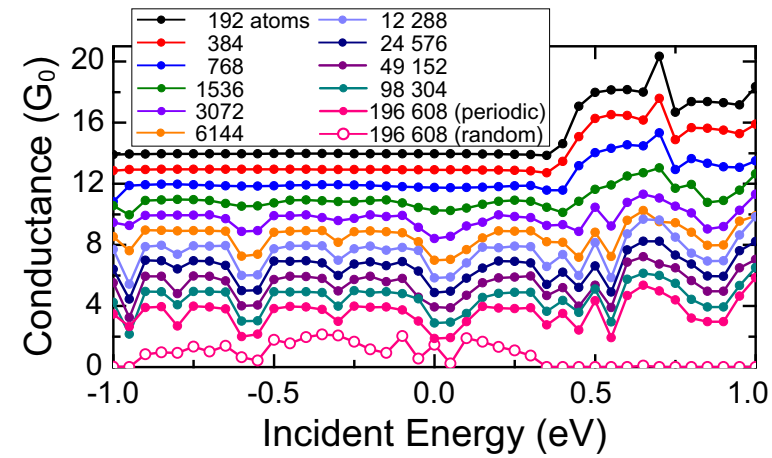

FIG. 4. Conductance spectra for BN-doped DWCNTs. The filled-circle and open-circle plots represent the spectra of the periodic and random models, respectively. The zero point of conductance is shifted by $1 \mathrm{G}_{0}$ between the adjacent spectra, where the vertical scale represents the value for the 196608 -atom models $(P=1024)$. The incident energy is measured from the Fermi level.

are notably reduced at the high incident energy because of the inter-states between the inner and outer tubes, as illustrated in Fig. 5(b). As $P$ increases, dips appear in the conductance spectra even at the low incident energy, and they gradually become prominent. This reflects the energy gap in the energy band structure that appears in an infinite DWCNT with a periodic scattering potential, as in the Kronig-Penney model. On the contrary, for the random model with $P=1024$ (196608 atoms, $\sim 1.0 \mu \mathrm{m}$ ), overall conductance is suppressed as plotted in Fig. 4. Although the transmission in most conduction channels is notably reduced due to the random scattering potential component widely distributed in DWCNT, there are still channels with high transmission to which the intra-states of the inner tube contribute. Here, the random model with $P=1024$ is prepared by randomly combining four different random models with $P=64$ and 96 . We also performed calculations using random models with different configurations, but the results are essentially the same. Performing the large-scale electron-transport calculation, we can discover this difference in the conductance spectra between the randomly and periodically doped models.

\section{CONCLUSION}

In summary, we developed an efficient first-principles algorithm for evaluating the electron-transport properties of long systems consisting of a vast number of atoms. The Green's function of the whole transition region extended towards the transport direction was obtained by recursively combining the Green's functions of the adjoining parts one by one. The computational cost for calculating the submatrices of the Green's functions required to estimate the transport properties is proportional to the number of the combined parts. As a practical application, we demonstrated the electron-transport calculations of BN-doped DWCNTs containing up to 196608 atoms. The proposed method develops our understanding of experimental studies on the research and development of devices using low-dimensional materials, as reported by Refs. [39-43]. Moreover, even in a submicron scale 2D and $3 \mathrm{D}$ structure containing millions of atoms, it is possible to 
(a)

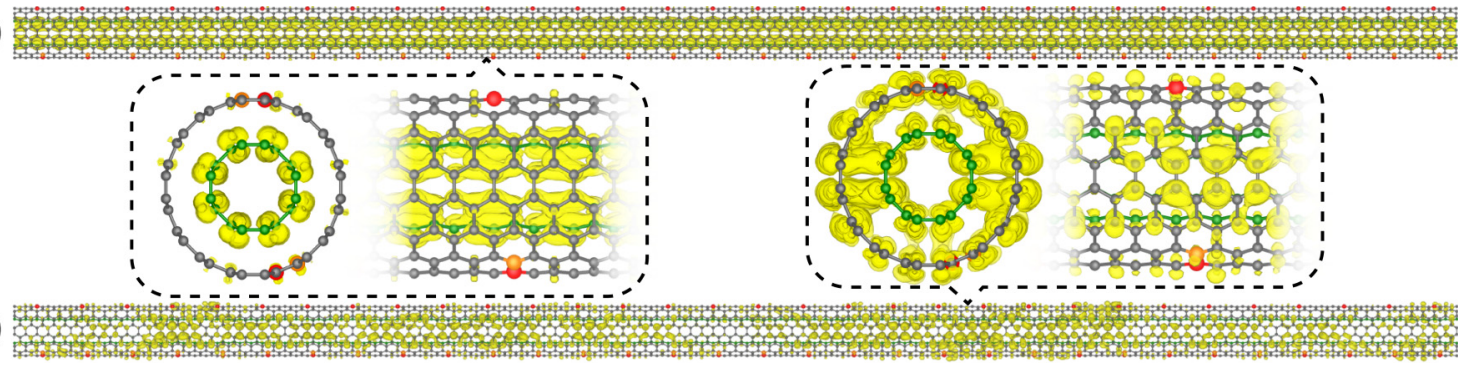

FIG. 5. Distributions of the scattering wave functions in the conduction channels of the 6144-atom model $(P=32)$. (a) and (b) illustrate the charge density of scattering wave functions with the highest transmission at the Fermi energy and $0.7 \mathrm{eV}$ above the Fermi energy, respectively. Insets surrounded by dashed lines depict cross-sectional and enlarged views. The key to the symbols is the same as that in Fig. 3 . The value of the yellow isosurface is $6.7 \times 10^{-4}$ electron $/ \AA^{3}$.

efficiently evaluate the electron-transport properties without any deterioration of computational accuracy by combining our previous algorithm [20] with the present method.

\section{ACKNOWLEDGMENTS}

This work was partially supported by the financial support from MEXT as a social and scientific priority issue (creation of new functional devices and highperformance materials to support next-generation industries) to be tackled by using post-K computer and JSPS KAKENHI Grants No. JP16H03865 and No. JP18K04873, and by MEXT as "Program for Promoting Researches on the Supercomputer Fugaku" (Quantum-Theory-Based Multiscale Simulations toward the Development of Next-Generation Energy-Saving Semiconductor Devices). S.T. acknowledges the financial support from Deutsche Forschungsgemeinschaft through the Projects No. 389895192 and No. 277146847. The numerical calculations were carried out using the system B and the system $\mathrm{C}$ of the Institute for Solid State Physics at the University of Tokyo, the Oakforest-PACS of the Center for Computational Sciences at University of Tsukuba, and the $\mathrm{K}$ computer provided by the RIKEN Advanced Institute for Computational Science through the HPCI System Research project (Project ID: hp190172).

\section{APPENDIX A: COMPUTATIONAL COST OF MATRIX-VECTOR OPERATION IN THE GREEN'S FUNCTION MATRIX CALCULATION}

As a demonstration, the number of the shifted conjugategradient (SCG) iterations $N_{\mathrm{SCG}}$ for calculating the subsets of the Green's function matrices is evaluated numerically using a double-walled carbon nanotube (DWCNT) system (see Fig. 3). For simplicity, we decide $N_{E}=1$ and the other conditions of the calculation were the same as those employed in Sec. III. Figure 6 plots the $N_{\text {SCG }}$ required to obtain the submatrices of the Green's function converged by the SCG method for the DWCNT system consisting of several unit cells. Here, the dimension of the $x$ and $y$ directions, $N_{x y}$, is fixed and the length of the DWCNT corresponding to $N_{z}$ is enlarged. It is confirmed that $N_{\mathrm{SCG}}$ increases linearly in proportion to $N_{z}$.

\section{APPENDIX B: INVERSE OF A 2 × 2 PARTITIONED MATRIX}

It is already known that the inverse of a $2 \times 2$ partitioned matrix is expressed as [44]

$$
\left[\begin{array}{l|l}
\mathbf{A}_{11} & \mathbf{A}_{12} \\
\hline \mathbf{A}_{21} & \mathbf{A}_{22}
\end{array}\right]^{-1}=\left[\begin{array}{c|c}
\mathbf{C}_{1}^{-1} & -\mathbf{C}_{1}^{-1} \mathbf{A}_{12} \mathbf{A}_{22}^{-1} \\
\hline-\mathbf{C}_{2}^{-1} \mathbf{A}_{21} \mathbf{A}_{11}^{-1} & \mathbf{C}_{2}^{-1}
\end{array}\right],
$$

where

$$
\begin{aligned}
& \mathbf{C}_{1}=\mathbf{A}_{11}-\mathbf{A}_{12} \mathbf{A}_{22}^{-1} \mathbf{A}_{21}, \\
& \mathbf{C}_{2}=\mathbf{A}_{22}-\mathbf{A}_{21} \mathbf{A}_{11}^{-1} \mathbf{A}_{12}
\end{aligned}
$$

are the so-called Schur's complement blocks. Note that both $\mathbf{A}_{11}$ and $\mathbf{A}_{22}$ must be invertible.

\section{APPENDIX C: DERIVATION OF $\mathcal{G}_{i: i+1}^{\mathrm{II}, \mathrm{UL}}$ AND $\mathcal{G}_{i: i+1}^{\mathrm{II}, \mathrm{UR}}$}

We first rewrite Eq. (4) into the following expression:

$$
E \mathbf{I}_{i}-\mathbf{H}_{i}-\left[\begin{array}{ccc}
\mathbf{0} & \mathbf{0} & \mathbf{0} \\
\mathbf{0} & . \cdot & \mathbf{0} \\
\mathbf{0} & \mathbf{0} & \boldsymbol{\Delta}_{1}
\end{array}\right]=\left[\mathcal{G}_{i: i+1}^{\mathrm{II}}\right]^{-1},
$$

where $\boldsymbol{\Delta}_{1}=\mathbf{B}_{i} \mathcal{G}_{i+1: i+1}^{\mathrm{UL}} \mathbf{B}_{i}^{\dagger}$ and is an $M_{i}$-dimensional square matrix. Here, we assume that the first block row and column have $N_{i-1}$ rows and columns, respectively. It is obvious that the last block row and column consist of $M_{i}$ rows and

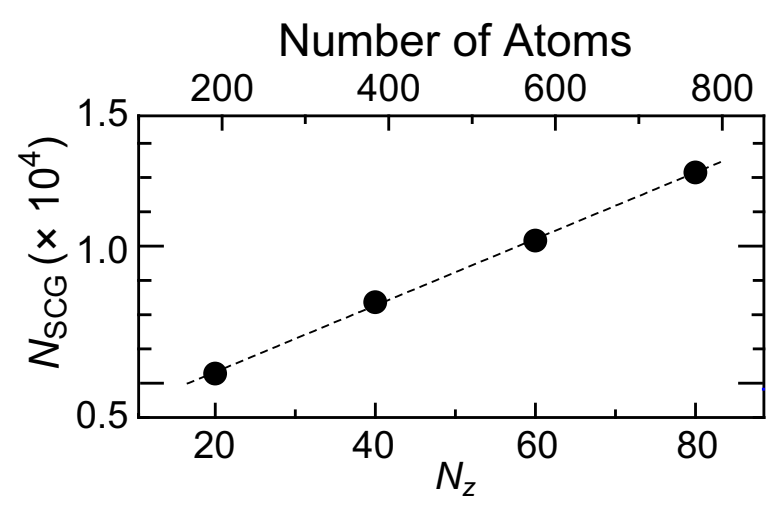

FIG. 6. The number of iterations, $N_{\mathrm{SCG}}$, required to obtain the subset of the Green's function matrices for DWCNTs converged by using the SCG method. 
columns, respectively. Operating $\mathcal{G}_{i: i+1}^{\text {II }}$ from the right and $\mathcal{G}_{i: i}=\left(E \mathbf{I}_{i}-\mathbf{H}_{i}\right)^{-1}$ from the left, one obtains

$$
\mathcal{G}_{i: i+1}^{\mathrm{II}}-\mathcal{G}_{i: i}\left[\begin{array}{ccc}
\mathbf{0} & \mathbf{0} & \mathbf{0} \\
\mathbf{0} & . \cdot & \mathbf{0} \\
\mathbf{0} & \mathbf{0} & \boldsymbol{\Delta}_{1}
\end{array}\right] \mathcal{G}_{i: i+1}^{\mathrm{II}}=\mathcal{G}_{i: i} .
$$

As discussed in the main text, we need only the submatrices located at the four corners of $\mathcal{G}_{i: i+1}^{\mathrm{II}}$. The submatrices satisfy the following $\left(N_{i-1}+M_{i}\right)$-dimensional linear equations:

$$
\mathbf{C}\left[\begin{array}{ll}
\mathcal{G}_{i: i+1}^{\mathrm{II}, \mathrm{UL}} & \mathcal{G}_{i: i+1}^{\mathrm{II}, \mathrm{UR}} \\
\mathcal{G}_{i: i+1}^{\mathrm{II}, \mathrm{LL}} & \mathcal{G}_{i: i+1}^{\mathrm{II}, \mathrm{LR}}
\end{array}\right]=\left[\begin{array}{ll}
\mathcal{G}_{i: i}^{\mathrm{UL}} & \mathcal{G}_{i: i}^{\mathrm{UR}} \\
\mathcal{G}_{i: i}^{\mathrm{LL}} & \mathcal{G}_{i: i}^{\mathrm{LR}}
\end{array}\right],
$$

where

$$
\mathbf{C}=\left[\begin{array}{cc}
\mathbf{I}_{N_{i-1}} & -\mathcal{G}_{i: i}^{\mathrm{UR}} \boldsymbol{\Delta}_{1} \\
\mathbf{0} & \mathbf{I}_{M_{i}}-\mathcal{G}_{i: i}^{\mathrm{LR}} \boldsymbol{\Delta}_{1}
\end{array}\right] .
$$

$\mathbf{C}^{-1}$ is analytically obtained using Appendix B as

$$
\mathbf{C}^{-1}=\left[\begin{array}{cc}
\mathbf{I}_{N_{i-1}} & \mathcal{G}_{i: i}^{\mathrm{UR}} \boldsymbol{\Delta}_{1}\left[\mathbf{I}_{M_{i}}-\mathcal{G}_{: i i}^{\mathrm{LR}} \boldsymbol{\Delta}_{1}\right]^{-1} \\
\mathbf{0} & {\left[\mathbf{I}_{M_{i}}-\mathcal{G}_{i: i}^{\mathrm{LR}} \boldsymbol{\Delta}_{1}\right]^{-1}}
\end{array}\right]
$$

Therefore the $N_{i-1}$-dimensional square submatrix located at the upper-left corner of $\mathcal{G}_{i: i+1}^{\mathrm{II}}$ is given as

$$
\begin{aligned}
\mathcal{G}_{i: i+1}^{\mathrm{II}, \mathrm{UL}}= & \mathcal{G}_{i: i}^{\mathrm{UL}}+\mathcal{G}_{i: i}^{\mathrm{UR}} \mathbf{B}_{i} \mathcal{G}_{i+1: i+1}^{\mathrm{UL}} \mathbf{B}_{i}^{\dagger} \\
& \times\left[\mathbf{I}_{M_{i}}-\mathcal{G}_{i: i}^{\mathrm{LR}} \mathbf{B}_{i} \mathcal{G}_{i+1: i+1}^{\mathrm{UL}} \mathbf{B}_{i}^{\dagger}\right]^{-1} \mathcal{G}_{i: i}^{\mathrm{LL}} .
\end{aligned}
$$

This is the analytical form of $\mathcal{G}_{i: i+1}^{\mathrm{II}, \mathrm{UL}}$.

The $\left(N_{i-1} \times M_{i}\right)$-dimensional submatrix located at the upper-right corner of $\mathcal{G}_{i: i+1}^{\text {II }}$ is written as

$$
\mathcal{G}_{i: i+1}^{\mathrm{II}, \mathrm{UR}}=\mathcal{G}_{i: i}^{\mathrm{UR}}\left(\mathbf{I}_{M_{i}}+\boldsymbol{\Delta}_{1}\left[\mathbf{I}_{M_{i}}-\mathcal{G}_{i: i}^{\mathrm{LR}} \boldsymbol{\Delta}_{1}\right]^{-1} \mathcal{G}_{i: i}^{\mathrm{LR}}\right) .
$$

Using one of the Searle's identities [45],

$$
\mathbf{I}-\mathbf{A}[\mathbf{I}+\mathbf{B A}]^{-1} \mathbf{B}=[\mathbf{I}+\mathbf{A B}]^{-1},
$$

one can transform Eq. (C7) into

$$
\mathcal{G}_{i: i+1}^{\mathrm{II}, \mathrm{UR}}=\mathcal{G}_{i: i}^{\mathrm{UR}}\left[\mathbf{I}_{M_{i}}-\mathbf{B}_{i} \mathcal{G}_{i+1: i+1}^{\mathrm{UL}} \mathbf{B}_{i}^{\dagger} \mathcal{G}_{i: i}^{\mathrm{LR}}\right]^{-1} .
$$

This is the analytic form of $\mathcal{G}_{i: i+1}^{\mathrm{II}, \mathrm{UR}}$.

APPENDIX D: DERIVATION OF $\mathcal{G}_{i: i+1}^{\mathrm{II}, \mathrm{LR}}$ and $\mathcal{G}_{i: i+1}^{\mathrm{II}, \mathrm{LL}}$

We first rewrite Eq. (6) into the following expression:

$$
E \mathbf{I}_{i+1}-\mathbf{H}_{i+1}-\left[\begin{array}{ccc}
\boldsymbol{\Delta}_{2} & \mathbf{0} & \mathbf{0} \\
\mathbf{0} & . \cdot & \mathbf{0} \\
\mathbf{0} & \mathbf{0} & \mathbf{0}
\end{array}\right]=\left[\mathcal{G}_{i: i+1}^{\mathrm{IV}}\right]^{-1},
$$

where $\boldsymbol{\Delta}_{2}=\mathbf{B}_{i}^{\dagger} \mathcal{G}_{i: i}^{\mathrm{LR}} \mathbf{B}_{i}$ and is an $N_{i}$-dimensional square matrix. Here, we assume that the last block row and column have $M_{i+1}$ rows and columns, respectively. It is obvious that the first block row and column consist of $N_{i}$ rows and columns, respectively. Operating $\mathcal{G}_{i: i+1}^{\mathrm{IV}}$ from the right and $\mathcal{G}_{i+1: i+1}=$ $\left(E \mathbf{I}_{i+1}-\mathbf{H}_{i+1}\right)^{-1}$ from the left, one obtains

$$
\mathcal{G}_{i: i+1}^{\mathrm{IV}}-\mathcal{G}_{i+1: i+1}\left[\begin{array}{ccc}
\boldsymbol{\Delta}_{2} & \mathbf{0} & \mathbf{0} \\
\mathbf{0} & . \cdot & \mathbf{0} \\
\mathbf{0} & \mathbf{0} & \mathbf{0}
\end{array}\right] \mathcal{G}_{i: i+1}^{\mathrm{IV}}=\mathcal{G}_{i+1: i+1} .
$$

As discussed in the main text, we need only the submatrices located at the four corners of $\mathcal{G}_{i: i+1}^{\mathrm{IV}}$. The submatrices satisfies the following $\left(N_{i}+M_{i+1}\right)$-dimensional linear equations:

$$
\mathbf{C}\left[\begin{array}{ll}
\mathcal{G}_{i: i+1}^{\mathrm{IV}, \mathrm{UL}} & \mathcal{G}_{i: i+1}^{\mathrm{IV}, \mathrm{UR}} \\
\mathcal{G}_{i: i+1}^{\mathrm{IV}, \mathrm{LL}} & \mathcal{G}_{i: i+1}^{\mathrm{IV}, \mathrm{LR}}
\end{array}\right]=\left[\begin{array}{ll}
\mathcal{G}_{i+1: i+1}^{\mathrm{UL}} & \mathcal{G}_{i+1: i+1}^{\mathrm{UR}} \\
\mathcal{G}_{i+1: i+1}^{\mathrm{LL}} & \mathcal{G}_{i+1: i+1}^{\mathrm{LR}}
\end{array}\right],
$$

where

$$
\mathbf{C}=\left[\begin{array}{cc}
\mathbf{I}_{N_{i}}-\mathcal{G}_{i+1: i+1}^{\mathrm{UL}} \boldsymbol{\Delta}_{2} & \mathbf{0} \\
-\mathcal{G}_{i+1: i+1}^{\mathrm{LL}} \boldsymbol{\Delta}_{2} & \mathbf{I}_{M_{i+1}}
\end{array}\right]
$$

$\mathbf{C}^{-1}$ is analytically obtained using Appendix B as

$$
\mathbf{C}^{-1}=\left[\begin{array}{cc}
{\left[\mathbf{I}_{N_{i}}-\mathcal{G}_{i+1: i+1}^{\mathrm{UL}} \boldsymbol{\Delta}_{2}\right]^{-1}} & \mathbf{0} \\
\mathcal{G}_{i+1: i+1}^{\mathrm{LL}} \boldsymbol{\Delta}_{2}\left[\mathbf{I}_{N_{i}}-\mathcal{G}_{i+1: i+1}^{\mathrm{UL}} \boldsymbol{\Delta}_{2}\right]^{-1} & \mathbf{I}_{M_{i+1}}
\end{array}\right] .
$$

Therefore the $M_{i+1}$-dimensional square submatrix located at the lower-right corner of $\mathcal{G}_{i: i+1}^{\mathrm{IV}}$ is given as

$$
\begin{aligned}
\mathcal{G}_{i: i+1}^{\mathrm{IV}, \mathrm{LR}}= & \mathcal{G}_{i+1: i+1}^{\mathrm{LR}}+\mathcal{G}_{i+1: i+1}^{\mathrm{LL}} \mathbf{B}_{i}^{\dagger} \mathcal{G}_{i: i}^{\mathrm{LR}} \mathbf{B}_{i} \\
& \times\left[\mathbf{I}_{N_{i}}-\mathcal{G}_{i+1: i+1}^{\mathrm{UL}} \mathbf{B}_{i}^{\dagger} \mathcal{G}_{i: i}^{\mathrm{LR}} \mathbf{B}_{i}\right]^{-1} \mathcal{G}_{i+1: i+1}^{\mathrm{UR}},
\end{aligned}
$$

This is the analytical form of $\mathcal{G}_{i: i+1}^{\mathrm{IV}, \mathrm{LR}}$.

The $\left(M_{i+1} \times N_{i}\right)$-dimensional submatrix located at the lower-left corner of $\mathcal{G}_{i: i+1}^{\mathrm{IV}}$ is given as

$$
\mathcal{G}_{i: i+1}^{\mathrm{IV}, \mathrm{LL}}=\mathcal{G}_{i+1: i+1}^{\mathrm{LL}}\left(\mathbf{I}_{N_{i}}+\boldsymbol{\Delta}_{2}\left[\mathbf{I}_{N_{i}}-\mathcal{G}_{i+1: i+1}^{\mathrm{UL}} \boldsymbol{\Delta}_{2}\right]^{-1} \mathcal{G}_{i+1: i+1}^{\mathrm{UL}}\right) .
$$

Using the Searle's identity (C8), one can transform Eq. (D7) into

$$
\mathcal{G}_{i: i+1}^{\mathrm{IV}, \mathrm{LL}}=\mathcal{G}_{i+1: i+1}^{\mathrm{LL}}\left[\mathbf{I}_{N_{i}}-\mathbf{B}_{i}^{\dagger} \mathcal{G}_{i: i}^{\mathrm{LR}} \mathbf{B}_{i} \mathcal{G}_{i+1: i+1}^{\mathrm{UL}}\right]^{-1} .
$$

This is the analytic form of $\mathcal{G}_{i: i+1}^{\mathrm{IV}, \mathrm{LL}}$.

[4] Y. Li, F. Qian, J. Xiang, and C. M. Lieber, Nanowire electronic and optoelectronic devices, Mater. Today 9, 18 (2006).

[5] F. Patolsky, G. Zheng, and C. M. L. Lieber, Nanowire-based biosensors, Anal. Chem. 78, 4260 (2006).

[6] Z.-M. Liao, Y.-D. Li, J. Xu, J.-M. Zhang, K. Xia, and D.-P. $\mathrm{Yu}$, Spin-filter effect in magnetite nanowire, Nano Lett. 6, 1087 (2006).

[7] V. V. Ivanovskaya, C. Köhler, and G. Seifert, $3 d$ metal nanowires and clusters inside carbon nanotubes: Structural, 
electronic, and magnetic properties, Phys. Rev. B 75, 075410 (2007).

[8] W. Kohn, Nobel Lecture: Electronic structure of matter-wave functions and density functionals, Rev. Mod. Phys. 71, 1253 (1999); P. Hohenberg and W. Kohn, Inhomogeneous electron gas, Phys. Rev. 136, B864 (1964); W. Kohn and L. J. Sham, Self-consistent equations including exchange and correlation effects, ibid. 140, A1133 (1965).

[9] T. Gunst, T. Markussen, M. L. N. Palsgaard, K. Stokbro, and M. Brandbyge, First-principles electron transport with phonon coupling: Large scale at low cost, Phys. Rev. B 96, 161404(R) (2017).

[10] F. Ducry, M. H. Bani-Hashemian, and M. Luisier, A Hybrid Mode-Space/Real-Space Scheme for DFT+NEGF Device Simulations, in 2019 International Conference on Simulation of Semiconductor Processes and Devices (SISPAD), Udine, Italy (2019), pp. 1-4.

[11] G. Calogero, N. Papior, M. Koleini, M. H. L. Larsen, and M. Brandbyge, Multi-scale approach to first-principles electron transport beyond $100 \mathrm{~nm}$, Nanoscale 11, 6153 (2019).

[12] H. Jippo, T. Ozaki, S. Okada, and M. Ohfuchi, Electronic transport properties of graphene channel with metal electrodes or insulating substrates in 10-nm-scale devices, J. Appl. Phys. 120, 154301 (2016).

[13] Y. Fujimoto and K. Hirose, First-principles treatments of electron transport properties for nanoscale junctions, Phys. Rev. B 67, 195315 (2003).

[14] K. Hirose, T. Ono, Y. Fujimoto, and S. Tsukamoto, FirstPrinciples Calculations in Real-Space Formalism (Imperial College Press, London, 2005).

[15] T. Ono, Y. Egami, and K. Hirose, First-principles transport calculation method based on real-space finite-difference nonequilibrium Green's function scheme, Phys. Rev. B 86, 195406 (2012).

[16] S. Iwase, T. Hoshi, and T. Ono, Numerical solver for first-principles transport calculation based on real-space finitedifference method, Phys. Rev. E 91, 063305 (2015).

[17] Y. Egami, S. Iwase, S. Tsukamoto, T. Ono, and K. Hirose, First-principles calculation method for electron transport based on the grid Lippmann-Schwinger equation, Phys. Rev. E 92, 033301 (2015).

[18] T. Ono and S. Tsukamoto, Real-space method for firstprinciples electron transport calculations: Self-energy terms of electrodes for large systems, Phys. Rev. B 93, 045421 (2016).

[19] S. Tsukamoto, T. Ono, K. Hirose, and S. Blügel, Self-energy matrices for electron transport calculations within the real-space finite-difference formalism, Phys. Rev. E 95, 033309 (2017); S. Tsukamoto, T. Ono, and S. Blügel, Improvement of accuracy in the wave-function-matching method for transport calculations, Phys. Rev. B 97, 115450 (2018); S. Tsukamoto, T. Ono, $\mathrm{S}$. Iwase, and S. Blügel, Complex band structure calculations based on the overbridging boundary matching method without using Green's functions, ibid. 98, 195422 (2018).

[20] Y. Egami, S. Tsukamoto, and T. Ono, Efficient calculation of self-energy matrices for electron-transport simulations, Phys. Rev. B 100, 075413 (2019).

[21] R. Takayama, T. Hoshi, T. Sogabe, S.-L. Zhang, and T. Fujiwara, Linear algebraic calculation of the Green's function for large-scale electronic structure theory, Phys. Rev. B 73, 165108 (2006).
[22] Although the computational cost of the subsets of the Green's function matrix is generally dominant, the cost of coefficient matrix-vector operations dominates in the case that $E$ becomes close to the eigenvalue of the truncated Hamiltonian matrix. The matrix-vector operation cost also becomes dominant when $N_{z}$ is large because the density of the eigenvalues in energy increases.

[23] D. J. Thouless and S. Kirkpatrick, Conductivity of the disordered linear chain, J. Phys. C: Solid State Phys. 14, 235 (1981).

[24] C. H. Lewenkopf and E. R. Mucciolo, The recursive Green's function method for graphene, J. Comput. Electron. 12, 203 (2013); P. Drouvelis, P. Schmelcher, and P. Bastian, Parallel implementation of the recursive Green's function method, J. Comput. Phys. 215, 741 (2006).

[25] D. K. Ferry and S. M. Goodnick, Transport in Nanostructures (Cambridge University Press, Cambridge, 1997), Chap. 3, p. 174.

[26] F. Sols, Recursive Tight-Binding Green's Function Method: Application to Ballistic and Dissipative Transport in Semiconductor Nanostructures, in Quantum Transport in Ultrasmall Devices, NATO Science Series: B Vol. 342, edited by D. K. Ferry, H. L. Grubin, C. Jacoboni, and A.-P. Jauho (Springer US, Boston, MA, 1995), pp. 329-338.

[27] G. Metalidis, Electronic Transport in Mesoscopic Systems, Ph.D. thesis, Martin-Luther-Universität Halle-Wittenberg, 2007.

[28] A. M. Souza, A. R. Rocha, A. Fazzio, and A. J. R. da Silva, Abinitio calculations for a realistic sensor: A study of $\mathrm{CO}$ sensors based on nitrogen-rich carbon nanotubes, AIP Adv. 2, 032115 (2012).

[29] M. Shin, W. J. Jeong, and J. Lee, Density functional theory based simulations of silicon nanowire field effect transistors, J. Appl. Phys. 119, 154505 (2016).

[30] W. Y. Rojas, C. E. P. Villegas, and A. R. Rocha, Ab initio modeling of spin relaxation lengths in disordered graphene nanoribbons, Phys. Chem. Chem. Phys. 21, 26027 (2019).

[31] In practical computation, we need not know the entire $\mathcal{G}_{i: i}$, only the submatrices located at the four corners.

[32] R. Courant and H. Robbins, What is Mathematics?: An Elementary Approach to Ideas and Methods, 2nd ed. (Oxford University Press, Oxford, England, 1996) p. 73.

[33] J. R. Chelikowsky, N. Troullier, K. Wu, and Y. Saad, Higherorder finite-difference pseudopotential method: An application to diatomic molecules, Phys. Rev. B 50, 11355 (1994); J. R. Chelikowsky, N. Troullier, and Y. Saad, Finite-DifferencePseudopotential Method: Electronic Structure Calculations without a Basis, Phys. Rev. Lett. 72, 1240 (1994).

[34] T. Ono and K. Hirose, Real-space electronic-structure calculations with a time-saving double-grid technique, Phys. Rev. B 72, 085115 (2005)

[35] K. Kobayashi, Norm-conserving pseudopotential database (NCPS97), Comput. Mater. Sci. 14, 72 (1999).

[36] N. Troullier and J. L. Martins, Efficient pseudopotentials for plane-wave calculations, Phys. Rev. B 43, 1993 (1991); L. Kleinman and D. M. Bylander, Efficacious Form for Model Pseudopotentials, Phys. Rev. Lett. 48, 1425 (1982).

[37] S. H. Vosko, L. Wilk, and M. Nusair, Accurate spin-dependent electron liquid correlation energies for local spin density calculations: A critical analysis, Can. J. Phys. 58, 1200 (1980). 
[38] H. Ishii, N. Kobayashi, and K. Hirose, Order- $N$ electron transport calculations from ballistic to diffusive regimes by a time-dependent wave-packet diffusion method: Application to transport properties of carbon nanotubes, Phys. Rev. B 82, 085435 (2010).

[39] A. D. Franklin and Z. Chen, Length scaling of carbon nanotube transistors, Nat. Nanotechnol. 5, 858 (2010).

[40] A. D. Franklin, M. Luisier, S.-J. Han, G. Tulevski, C. M. Breslin, L. Gignac, M. S. Lundstrom, and W. Haensch, Sub-10 nm carbon nanotube transistor, Nano Lett. 12, 758 (2010).

[41] C. Qiu, Z. Zhang, M. Xiao, Y. Yang, D. Zhong, and L.-M. Peng, Scaling carbon nanotube complementary transistors to 5-nm gate lengths, Science 355, 271 (2017).
[42] Y. Sun, Z. Peng, H. Li, Z. Wang, Y. Mu, G. Zhang, S. Chen, S. Liu, G. Wang, C. Liu, L. Sun, B. Man, and C. Yang, Suspended CNT-Based FET sensor for ultrasensitive and label-free detection of DNA hybridization, Biosens. Bioelectron. 137, 255 (2019).

[43] M. E. Schmidt, M. Muruganathan, T. Kanzaki, T. Iwasaki, A. M. M. Hammam, S. Suzuki, S. Ogawa, and H. Mizuta, Dielectric-screening reduction-induced large transport gap in suspended sub-10 nm graphene nanoribbon functional devices, Small 15, 1903025 (2019).

[44] K. B. Petersen and M. S. Pedersen, The Matrix Cookbook (2012), http://www2.imm.dtu.dk/pubdb/p.php?3274.

[45] S. R. Searle, Matrix Algebra Useful for Statistics (Wiley, New York, NY, 1982), Chap. 5, p. 151. 